\title{
PEIRCE Y LA LITERATURA: EL ESTADO DE LA CUESTIÓN
}

\author{
Lucia Santaella Braga
}

(Universidad Católica de São Paolo)

Aunque no esté explícitamente ligado a la cuestión de la literatura, «En Busca de la Esencia del Lenguaje» (Jakobson 1970) se constituye en ensayo seminal, verdadera puerta de entrada para aquellos que se interesan por el tema «Peirce y la Literatura». Lingüista dotado de sutilísima sensibilidad para los fenómenos de poeticidad de la lengua, Jakobson fue el primero, de que tengo noticia, en percibir la importancia de los tres tipos de signos (iconos, índices, símbolos), elaborados por Peirce, para cuestionar la tesis de la arbitrariedad de los signos linguísticos. Con elegante argumentación y abundancia de ejemplos, «En Busca de la Esencia del Lenguaje» comprueba la presencia de los aspectos icónicos e indiciales en el seno del lenguaje verbal, presumidamente simbólico, así como discute el papel fundamental y motivador desempeñado por los iconos e índices en la construcción del sentido. No es necesario insistir en la importancia de esos factores para la comprensión del lenguaje literario, más específicamente de la poesía.

Obra pionera, al enfocar directamente algunas de las relaciones posibles entre Peirce y la Literatura, es Semiótica y Literatura, de Décio Pignatari (1974). Ese trabajo, defendido originalmente como tesis de 
doctorado, en 1973, es fruto, sobre todo, de una lectura inspirada en Peirce. Desde la propuesta de un cuasimétodo, metamétodo heurísticosemiótico (Valéry-Peirce), pasando por la concepción de una proto-estética extraída de la fenomenología y de las clasificaciones de signos peirceanos, hasta las «descifraciones semióticas» de algunos textos literarios seleccionados, se trata de una obra que revela una opción por la interpretación creativa de algunos de los textos de Peirce, en detrimento de la mera exégesis textual.

Digno de destacar, por la belleza y poeticidad teórica, es su tratamiento de la «cuestión del icono», que se extiende del «breve gesto a una teoría del casi-signo», tan breve como admirablemente sensible y exacto.

En el contexto de los Estados Unidos, John K. Sheriff es el autor que más se ha dedicado a la relación entre Peirce y la Literatura. En 1981, publicó un artículo titulado «Charles S. Peirce and the Semiotics of Literature», que tenía por objetivo «revelar que la teoría de los signos desarrollada por el contemporáneo americano de Saussure, Charles S. Peirce, proporciona un marco de referencias que permitiría a los semióticos ver más allá de las limitaciones del análisis saussureano del signo, esclareciendo muchas cuestiones que han sido problemáticas en la semiótica de la literatura» (: 51). En 1989, Sheriff amplió las ideas expuestas en ese artículo, publicando el libro The Fate of Meaning, con el siguiente subtítulo Charles Peirce, Structuralism and Literature. En palabras del autor, las tesis desarrolladas en el libro son las siguientes:

\footnotetext{
... las cuestiones que preocuparon a teóricos de la literatura - cuestiones de la intención del autor, la autonomía del texto, el papel del lector, la naturaleza del lenguaje, la fuente y la naturaleza del significado- tienen respuestas bien diferentes, dependiendo de la definición de signo de la que partimos. Mi primera tesis es que, si usted va en busca del sentido y parte de una definición diádica, saussureana, del signo, «usted no podrá llegar allá, desde aquí» (...) Mi segunda tesis es que, si usted comienza su búsqueda del sentido con una definición peirceana del signo, «usted no se perderá en la búsqueda con tal de que prosiga en ella» (: XVIII)
}

Los principales argumentos en los que las tesis del autor se desarrollan están en los siguientes capítulos: «Arte: el sentido como un signo de posibilidad», «Crítica: el sentido como un signo de hecho» y «Teoría: el sentido como un signo de razón».

Los títulos de los capítulos, como se puede ver, son sugestivos. Desgraciadamente, sin embargo, prometen más de lo que cumplen, ya que, en ellos, el autor gasta mucho más tiempo y palabras en comparar algunas de las ideas de Peirce con las de otros autores, principalmente de Derrida y de Wittgenstein, por ejemplo, que en explorar adecuada- 
mente las nociones peirceanas, con toda la profundidad que el lector debía esperar.

Es, no obstante, en los textos de otro autor, el dinamarqués Jorge Dines Johansen, donde vamos a encontrar esa esperada profundidad y minucia en el trabajo sobre los escritos de Peirce aplicados al campo de la literatura. Johansen es uno de los más conocidos y respetados especialistas en Peirce, en el contexto internacional. A partir de un conocimiento seguro, afianzado por las consultas cuidadosas incluso de los manuscritos no publicados de Peirce, Johansen ha ido creando a lo largo de los años una teoría de la literatura basada en las doctrinas peirceanas. Para ello, el autor realiza aquello que, a mi juicio, constituye el procedimiento estratégicamente más eficaz, cuando se trata de aplicar Peirce a algún campo de semiosis específica. Se hacen las conexiones y mediaciones explicatorias necesarias a la vinculación entre los fundamentos peirceanos generales y abstractos y las restricciones particulares impuestas por un tipo peculiar de funcionamiento de los signos, en este caso, el de la literatura.

Dentro del área de la interpretación de los textos en general, y del texto literario en particular, el extenso artículo de Johansen, «Prolegomena to a semiotic theory of text interpretation» (1985) se configura como uno de los ejemplos más perfectos, por un lado, por la paciente dedicación a la tarea interpretativa en torno a Peirce; por otro lado, por la creación de las transiciones intermedias, necesarias para el paso de la generalidad de los conceptos hasta el nivel de mayor concreción de una teoría más específica, relativa a la semiosis literaria en particular.

También de Johansen, otros trabajos dignos de mención, igualmente relativos al tema "Peirce y la Literatura», son: «The Place of Semiotics in the Study of Literature» (1986), «What is a Text? Semiosis and Textuality, a Peircean Perspective» (1988) y «Hypothesis, Reconstruction, Analogy: On Hermeneutics and the Interpretation of Literature» (1989).

«The Realism of C. S. Peirce, or how Homer and Nature can be the same», de Joel Weinsheimer (1983), es otro estudio igualmente capaz de enraizar las implicaciones de una posible teoría de la literatura peirceana en el contexto filosófico-conceptual que da significado a las clasificaciones de signos. Discutiendo la semiótica como sólo uno de los aspectos de la filosofía realista de Peirce, Weinsheimer presenta el modo por el cual «los críticos pueden establecer la función referencial de la literatura, sin minimizar las relaciones intertextuales que aparentemente sugieren la negación de la referencia» (: 225 ). El texto hace, en síntesis, una brillante defensa del realismo, como una de las consecuencias más cruciales de la teoría peirceana de los signos para nuestro entendimiento de la literatura y del arte. Según nos indica V. Colapietro (1989: 12), Weinsheimer expo- 
ne con claridad ejemplar que «el reconocimiento del carácter esencialmente icónico de una obra de arte no necesita pagar el precio de la negligencia de su aspecto indicial o simbólico».

\section{TEORÍA Y PRÁCTICA}

Buscando un equilibrio entre la discusión teórica y la aplicación práctica de los conceptos de Peirce, se encuentra el libro The Reading of Time. A Semantico-Semiotic Approach, de Julio Pinto (1989). Aunque el título del libro sugiere amplitud, su intención es más bien limitada: en el contexto de análisis de la narrativa literaria, el autor enfoca las estrategias interpretativas del lector relativas a las relaciones temporales. Si no se puede negar que el libro gana en términos de pretendido equilibrio entre teoría y práctica analítica, se debe observar, a pesar de ello, que esto se realiza a costa de una innegable fragmentación y cierto esquematismo a que es sometida la teoría peirceana. La lectura de los textos literarios, al final del libro, no obstante, presenta análisis bastante instructivos.

Una aplicación de las categorías fenomenológicas a la interpretación de un personaje literario es lo que Dinda L. Gorlée (1987) presenta en su artículo «Firstness, Secondness, Thirdness and Cha(u)nciness». En palabras de la autora, los objetivos de esta aplicación serían: «La primeridad icónica, la segundidad indexical y la terceridad simbólica, como estadios de los procesos de aprendizaje humano, nos servirán como punto de partida teórico para la evaluación del modo en el que el protagonista de la novela Being There, de Jerzy Kosinski, experimenta el mundo». El análisis efectuado por Gorlée es perspicaz y lleno de humor inteligente.

Además de su tesis de doctorado inédita, «Manoel Bandeira e a Poética do Elemento Lexical» (1973), Lucia Santaella Braga contribuyó al tema de Peirce y la Literatura con dos artículos. El primero de ellos, «Entre-ver la literatura, Inter-leyendo un poema» (1981), confronta, de modo brevísimo, la semiótica peirceana y la semiología europea, para terminar proponiendo la lectura semiótica de un poema. El segundo artículo (1980, con traducción al inglés en 1990), más ambicioso en sus objetivos y más complejo en sus implicaciones, crea una clasificación de nueve tipos de textos verbales-escritos, con base en las categorías fenomenológicas y en las tríadas peirceanas. Algunos de estos tipos de textos son específicamente literarios, otros son extensivos a la literatura.

También dirigido hacia una tipología, pero ésta relativa a los interpretantes, se encuentra el artículo de Mariana Net, «Types d'interpretants du texte artistique - reférent, symbole, topos, motif» (1991). Aplicando 
el proceso de semiosis a la recepción de textos artísticos, la autora trabaja con varios modelos de interpretación de textos que terminan en el análisis de funciones de los emblemas, tanto en el texto artístico cuanto en el interior de un espacio cultural más vasto.

Contribución bastante importante para la construcción de una estética informacional, con fuerte inspiración peirceana, es la obra Pequeña Estética, de Max Bense (1971). Estando sus cuestiones más directamente ligadas a la estética, la mención de este libro no debería, en rigor, aparecer en este punto, y sí en la presentación que reservé, al final, para todo un grupo de textos que se refieren específicamente a la estética peirceana. Entretanto, por lo menos en la edición brasileña del libro a la que me estoy refiriendo, hay una segunda parte dedicada específicamente a la poesía, artes visuales, etc., lo que justifica la inserción de Bense en este lugar dedicado a la presentación de los textos, secuencia cuya lógica probablemente quedará clara al lector, al final de estos comentarios.

\section{LA POÉTICA DE LA TRADUCCIÓN}

Todavía con inspiración peirceana, pero dedicados a los problemas de la traducción creativa, encontramos los artículos de Haroldo de Campos. Desde 1967, con su ensayo «Da tradução como criação e como critica», este poeta, traductor, crítico y teórico de la literatura viene desarrollando, en Brasil, a partir de su prolífera y admirable práctica de poeta-traductor, una teoría de la traducción concebida como re-creación, esto es, traducción como signo de invención, o «transcreación», tal como Haroldo de Campos la bautizó. Son innumerables los artículos que el autor publicó sobre ese asunto, a lo largo de más de veinte años. A partir de finales de los años 70, e inicios de los 80, sin embargo, el trinomio BenjaminValéry-Peirce se convirtió en el campo de referencia más nítido de su teoría. Destacan a este respecto los siguientes artículos: «Tradução, Fantasia, Fingimento» (1983) y «Para além do principio da saudade» (1984).

También en el ámbito de la teoría y práctica de la traducción creativa, extendida ahora al terreno de las artes, medias y literatura en general, e intensamente fundamentada en la semiótica peirceana, se encuentra el libro Tradução Intersemiótica, de Julio Plaza (1987). El rasgo más original de ese trabajo está en la apropiación peculiar que el autor hace de la teoría de los signos, esto es, una apropiación diagramática. Lejos de funcionar como camisa de fuerza reductora de la ambiguiedad de los fenómenos examinados, la teoría es, al contrario, utilizada como base que va orientando 
la visión de la traducción desde su nivel macro («traducción como pensamiento en signos, como intercurso de los sentidos, como transcreación de las formas»), hasta los niveles microscópicos de los tránsitos y operaciones sígnicas que se procesan en el interior de un acto traductor.

\title{
EL TEMA DE LA METÁFORA
}

Presentado esto, podemos pasar ahora a la discusión de todo un grupo de obras dedicadas al tema de la metáfora basadas en las teorías de Peirce. El libro más antiguo, que inicia el tratamiento de este tema, debe ser Lenguage, Thought, and Culture, de Paul Henle (1958). Sobre el tipo de enfoque que Henle dio a la metáfora, transcribo aquí los comentarios de Carl R. Hausman, en la actualidad uno de los más autorizados especialistas en el asunto. Mencionando los intentos, realizados por Henle, de desarrollar una visión de la metáfora influenciado por la distinción peirceana entre símbolo e icono, Hausman (1989: 218-19), dice:

\begin{abstract}
Henle propone la idea de que las metáforas contienen elementos icónicos. No obstante, prescinde de la distinción peirceana entre la segunda y tercera especies de iconos, esto es, entre los iconos analógicos y los iconos metafóricos. Consecuentemente, Henle trata la visión de Peirce como si éste concibiese las metáforas como analogías. Aparentemente, la concepción del propio Henle, sobre como las metáforas pueden revelarse inteligibles, ya estaba tan fuertemente fijada que él no consiguió captar las sutilezas de la visión peirceana. Más grave que esto es el hecho de que Henle no se haya interesado por el modo cómo los iconos se refieren a los objetos (...)
\end{abstract}

Famosos, evidentemente, son los trabajos de Umberto Eco sobre la metáfora, que aparecen en The Role of the Reader (1979) y, más específicamente, en Semiotics and the Philosophy of Language (1984). No obstante, los estudios de Eco están más dirigidos hacia una semántica de la metáfora que basados en la semiótica de Peirce. De éste, sólo son perceptibles algunas resonancias esporádicas de los conceptos (incuestionablemente peirceanos) de abducción e interpretante.

Pero determinantemente basados en Peirce están los libros Metaphor Reexamined, de Lisolette Gumpel (1984), y el de Michael y Marianne Shapiro (1988) Figuration in Verbal Art. Para el reexamen y propuesta de una concepción no-aristotélica de la metáfora, L. Gumpel utiliza las tricotomías peirceanas (1. cualisigno, icono, y rema; 2 . sinsigno, índice y dicente; 3 . legisigno, símbolo y argumento), y con énfasis en el polo del interpretante: rema, dicente o proposición y argumento. El libro de los 
Shapiro, a su vez, es parte de un proyecto de mayor envergadura que viene siendo realizado por Michael, desde hace algunos años, y que apunta al desarrollo de una lingüística peirceana. Ex-discípulo de Jakobson, Michael Shapiro está llevando adelante la sugerencia del maestro de que una transformación puede suceder en el momento en que la lingüística descubra y adopte las consecuencias de la semiótica de Peirce para el estudio del lenguaje verbal. De hecho, el proyecto de Shapiro ha influido en algunos lingüistas norteamericanos que, con entusiasmo, están aliándose a la tarea.

El libro de Michael Cabot Haley (1988), The Semiosis of Poetic Metaphor, por ejemplo, nació directamente bajo el impacto de los efectos que el contacto con Shapiro produjo en Haley. Por tratar específicamente de la metáfora poética, su libro es de sustancial importancia dentro del tema Peirce y la Literatura. Así se expresa el autor con relación a sus objetivos:

\footnotetext{
Con la pretensión de funcionar como un paso preliminar al desarrollo de una teoría (orgánicamente holística y rigurosamente analítica) de la metáfora poética, este estudio sólo aplica la más famosa tricotomía peirceana de tipos de signos (el símbolo, el índice, el icono), así como su, rara vez, recordada, tricotomía de hipoiconos, a algunos rasgos estéticos y semánticos de la metáfora poética que son frecuentemente ignorados (: 6)
}

Ampliando el área a una teoría de la metáfora en general, el más ambicioso y respetable estudio perceptiblemente basado en Peirce, en especial en su epistemología sígnica, es el libro Metaphor and Art. Interactionism and Reference in the Verbal and Nonverbal Arts, de Carl R. Hausman (1989).

Una de las principales preocupaciones del libro nació de la convicción del autor de que «el modo cómo las palabras u otras unidades lingüísticas mayores funcionan dentro de las metáforas $\mathrm{y}$, consecuentemente, dentro del contexto de las metáforas, es comparable a las funciones de los componentes de las artes visuales y de la música» (: IX). Con esa convicción en mente, el libro desarrolla «una serie de especulaciones sistemáticas y críticas sobre las funciones del lenguaje metafórico, las artes y la realidad». Para eso, el autor ofrece «un retrato de cómo las expresiones metafóricas funcionan con relación al mundo» $(: 10)$. El aspecto más relevante del libro e indiscutiblemente más en deuda con Peirce, está, sin duda, en aquello que Hausman denomina «ontología de la metáfora», que así se expresa:

Además de examinar la estructura de la metáfora, también analizaré cómo las metáforas se relacionan con la realidad. Al considerar esta cuestión, me concentraré más en la referencia que en el sentido o significado connotativo de la metáfora. La 
referencia, así como el sentido y el contenido, además de la forma, son necesarios para dar a las metáforas su significancia y valor. Esta parte de la discusión necesariamente se vuelve hacia la ontología. Mi proposito principal será el de esquematizar aquello que una ontología necesita ser, y sugerir cómo afecta al modo en que las metáforas y las obras de arte, a su vez, pueden ser entendidas (:11)

De gran importancia, para aquellos que se interesan por los fundamentos peirceanos de la obra de Hausman, es el apéndice «Metaphorical Reference and Peirce's Dynamical Object», cuyo título es, por sí mismo, capaz de poner en evidencia que las fuentes de la ontología, desarrollada por el autor, fueron buscadas en la teoría peirceana del objeto del signo.

\section{ABDUCCIÓN Y CREATIVIDAD}

Otro grupo de obras de extrema relevancia para la investigación literaria son los estudios sobre creatividad. Por ahora, hablar de creatividad bajo una óptica peirceana es hablar de la forma de cuasi-raciocinio, llamada abducción, el más notable y notorio entre los innumerables descubrimientos teóricos de Peirce.

La lógica abductiva como medio para la comprensión de los procesos de creación en la literatura, especialmente en el texto poético, se configura como la espina dorsal del libro de Ángel Herrero (1988), Semiótica y Creatividad - La Lógica Abductiva. Además de la abducción, el autor trabaja con varios conceptos que están implicados en los procesos abductivos: las categorías fenomenologicas (primeridad, segundidad, terceridad), semiosis, icono. De esos conceptos extrae consecuencias originales sobre «el carácter semiótico del reconocimiento icónico», «la iconicidad abductora de la metáfora», etc., que se amplían hasta las consideraciones acerca de una estética peirceana. El libro es muy denso y complejo, no sólo en los tópicos que aborda, sino también en el número de autores y respectivas teorías que maneja para la elaboración de sus ideas. Como ya mencioné en un artículo en el que reseñé el libro de Herrero (Santaella Braga 1991), el autor parece tropezar un poco en los conceptos peirceanos. No por eso deja de extraer de la abducción las consecuencias posibles.

También relativo a la creatividad y, para ello, haciendo uso igualmente del concepto de abducción, encontramos el artículo de F. Scott (1983), «Process from the Peircean point of view: some aplications to art». Como el título sugiere, el eje central del artículo se desarrolla en torno al concepto de proceso. Éste, no obstante, es aclarado en conjun- 
ción con la abducción como factor sobresaliente en la interpretación de las obras de arte.

El libro de Douglas R. Anderson (1987), Creativity and the Philosophy of C. S. Peirce, también comienza con la abducción para explicar la creatividad en la ciencia y, posteriormente en el arte, pero amplía el tema a la evolución creativa en el ámbito de la metafísica peirceana. El libro es coherente y bien estructurado, pero peca por la excesiva carga valorativa que coloca sobre el agapismo (evolución por amor creativo) que, de acuerdo con Peirce, apenas representa uno de los tipos posibles de evolución. Para aquellos que estén interesados por una fundamentación filosófica más equilibrada del concepto de crecimiento creativo, cumple indicar el artículo de Sandra B. Rosenthal (1989), «Processive Emergence and the Peircean Categories».

Las obras aquí mencionadas, relativas a la cuestión de la abducción y creatividad, ciertamente no agotan, con mucho, la bibliografía existente sobre la abducción peirceana. La literatura relativa a esto es hoy voluminosa, especialmente porque el concepto de abducción está volviéndose moneda corriente entre los especialistas en inteligencias artificiales y los cognitivistas en general. La tendencia es la de que la circulación intelectual del término supere, si es que ya no se ha superado, la famosa tríada de icono, índice, símbolo. Para recoger una parcela representativa de la bibliografía sobre abducción, sería necesario un estado de la cuestión mucho más extenso que el presente. Con todo, como no trato aquí de la abducción en general, me he limitado a citar los trabajos que directa o indirectamente relacionan la abducción con la literatura y el arte.

\section{LA ESTÉTICA PEIRCEANA}

Con eso, pienso que podemos pasar ahora al último grupo de obras que fueron seleccionadas para figurar en la reseña bibliográfica relativa a Peirce y la Literatura. Aunque no esté directamente ligada a este tema, la estética peirceana constituye, a mi modo de ver, un fundamento que no puede ser ignorado por las investigaciones literarias de base peirceana. Justifico, así, una breve mención de los textos que han sido más citados. Son los siguientes: Max O. Hocutt (1962), C. M. Smith (1972), B. Kent (1976), J. Jay Zeman (1977), E. F. Kaelin (1980), Kim Smith (1983), B. Kent (1987) y Herman Parret (1990).

Todos los textos son unánimes en la constatación de la extrema originalidad y alto contenido sugestivo de las concepciones, algo dispersas, 
pero no por eso asistemáticas, que Peirce desarrolló sobre estética. Siendo ésta la primera entre las ciencias normativas (seguida por la ética $\mathrm{y}$, subsecuentemente, por la lógica o semiótica), su clave de desciframiento se encuentra en la primera categoría fenomenológica (primeridad o posibilidad, presentidad, frescor, originalidad, espontaneidad, fragilidad, calidad, sentimiento, etc.), así como en los signos correspondientes a esa categoría (cualisigno, icono, rema). Todos los textos trabajan, cada uno a su modo, con esos conceptos en un intento de comprender la «calidad de sentimiento» propia del efecto estético. El texto más completo y más diferenciado con relación a los demás es el de $\mathrm{B}$. Kent (1987), una vez que la autora incluye la estética en el vasto diagrama peirceano de las ciencias, proporcionando datos inéditos para su entendimiento.

No me parece necesario decir que la lista de los textos, que fueron citados en este estado de la cuestión, no tiene la pretensión de ser exhaustiva. Hay un elevado número de revistas de semiótica, publicadas en las más diversas lenguas, en un gran número de países del mundo. Conocer el contenido de cada una de esas revistas, para verificar si hay artículos de semiótica literaria basados en Peirce, no es tarea para un solo investigador. Las obras aquí citadas son aquellas que más han circulado y que, por eso mismo, se han hecho más conocidas. Si no son suficientes, son por lo menos representativas para quien pretenda entrar en el elenco de aquellos que se han consagrado a los estudios de Peirce y la Literatura. A ésos, que sean bienvenidos. A los que ya están incluidos en este elenco, pero aquí involuntariamente olvidados, dentro de la lógica de la certeza anticipada, presento, de antemano, mis disculpas.

\section{Referencias bibliográficas}

Anderson D. R. (1987): Creativity and the Philosophy of C. S. Peirce. Dordrecht: Martinus Nijhoff Publishers.

Bense, M (1971): Pequeña Estética. São Paulo: Perspectiva.

CAMPOS, H. DE (1967). «Da tradução como criação e como critica». En Metalinguagem, Petrópolis: Vozes, 21-28.

- (1983): «Tradução, Fantasia e Fingimento». Folhetim 348, Folha de São Paulo.

- (1985): «Para além do principio da saudade». Folhetim 412, Folha de São Paulo.

COLAPIETRO, V. (1989): «Book Review». Society for the Advancement of American Philosophy. Newsletter 54, 10-12.

Eco, U. (1979): The Role of the Reader. Bloomington: Indiana University Press.

- (1984): Semiotics and the Philosophy of Language. Bloomington: Indiana University Press. 
GoRLÉE, D. L. (1987): «Firstness, Secondness, Thirdness and Cha(u)nciness». Semiotica $65-1 / 2,45-55$.

Gumpel, L. (1984): Metaphor Reexamined. A Non-Aristotelian Perspective, Bloomington: Indiana University Press.

Haley, M. C. (1989): The Semiosis of Poetic Metaphor. Bloomington: Indiana University Press.

HAUSMAN, C. R. (1989): Metaphor and Art. Interactionism and Reference in Verbal and Nonverbal Arts. Cambridge Univsersity Press.

Henle, P. (1958): Language, Thought, and Culture. Ann Arbor: University of Michigan Press.

Herrero, A. (1988): Semiótica y Creatividad. La Lógica Abductiva, Madrid: Palas Atenea.

HocuTT, M. O. (1962): «The Logical Foundations of Peirce's Aesthetics». Journal of Aesthetics and Art Criticism 21, 157-166.

JAKOBSON, R. (1970): «A procura da essencia da linguagem». En Linguística $e$ Comunicação. São Paulo: Cultrix, 98-117.

JohANSEN, J. D. (1985): «Prolegomena to a Semiotic Theory of Text Interpretation». Semiotica 57-3/4, 225-288.

- (1986): «The Place of Semiotics in the Study of Literature». En Semiotica and International Scholarship: Towards a Language or Theory, Jonathan D. Evans y André Helbo (eds.). Dordrecht: Martinus Nijhoff, 101-126.

- (1988) «What is a Text? Semiosis and Textuality, A Peircean Perspective» Livstegn $5 / 1,7-32$.

- (1989): «Hypothesis, Reconstruction, Analogy: On Hermeneutics and the Interpretation of Literature». Semiotica 74-3/4, 235-252.

KaElin, E. F. (1980): «Reflections on Peirce's Aesthetics». The Monist vol. 63, n. 3 , 142-155.

KENT, B. (1976): «Peirce's Esthetics: A New Look». Transactions of the Charles $S$. Peirce Society 12, 263-283.

- (1987): Charles S. Peirce. Logic and the Classification of the Sciences. Kingston and Montreal: McGill-Queen's University Press.

NET, M. (1991): «Types d'interpretants du text artistique reférent, simbole, topos, motif». En Semiotik Interdisziplinär II, Jeff Bernard (Hg.), Wien: OGS, 35-46.

PARret, H. (1990): «Fragmentos Peirceanos sobre a Experiencia Estética». FACE 3, 2, 217-228.

Pignatari, D. (1974): Semiótica e Literatura. São Paulo: Perspectiva.

PINTo, J. (1989): The Reading of Time. A Semantico-Semiotic Approach. Berlín: Mouton de Gruyter.

PlazA, J. (1987): Tradução Intersemiótica. São Paulo: Perspectiva.

Rosenthal, S. (1989): «Processive Emergence and the Peircean Categories». VS 49, 121128.

SANTAElla Braga, L. (1973): «Manuel Bandeira e a Poética do Elemento Lexical», tesis doctoral, PUCSP.

- (1980): «Para uma classificação da linguagem escrita», En Produção e Liguagem $e$ Ideologia. São Paulo: Cortez, 143-160.

- (1981): «Entrevendo a Literatura, Interlendo um poema». DeSignos 6, 52-61.

- (1990): «For a classification of written language». Acciones Textuales 1, 55-74.

- (1991): «Instinct, Logic or the Logic of Instinct? Semiotica 83-1/2, 123-141.

Shapiro, M. y Shapiro, M. (1988): Figuration in Verbal Art. Princeton: Princeton University Press. 
SHERIFF, J. K. (1981): «Charles S. Peirce and the Semiotics of Literature». En Semiotic Themes, R. T. DeGeorge (ed.). Lawrence: University of Kansas Publication.

- (1989): The Fate of Meaning. Charles Peirce, Structuralism and Literature. Princeton: Princeton University Press.

SMith, C. M: (1972): «The Aesthetics of Charles S. Peirce». Journal of Aesthetics and Art Criticism 31, 21-29.

Smith, K. (1983): «Peirce and the Prague School on the Foundational Role of the 'Aesthetic' Sign». American Journal of Semiotics, 2, 1-2, 175-194.

Weinsheimer, J. (1983): «The Realism of Charles S. Peirce or How Homer and Nature can be the same». American Journal of Semiotics, 2, 1-2, 225-264.

ZEMAN, J. J. (1977): «The Esthetic Sign in Peirce's Semiotic». Semiotica 19-3/4, 241258. 